\title{
Audit and feedback for individual practitioners in the emergency department: an evidence-based and practical approach
} \begin{abstract}
$\mathrm{MD}^{\S \pi ;}$ Edmund Kwok, MD\#; Eddy Lang, MDCM*
\end{abstract}
Shawn K. Dowling (D), MD*; Shawn Mondoux (D), MD ${ }^{\dagger \neq}$; Christopher M. Bond, MD*; Amy H.Y. Cheng, practice gaps. Furthermore, the Competency by Design physicians to understand and assess their practice, create and implement a learning plan and evaluate the out-

\section{What is audit and feedback? Why is it needed?}

Audit and feedback is a practice improvement strategy. By summarizing an individual's clinical performance over a specific time period, it allows for the identification of areas for improvement. The rationale is that, by demonstrating a gap between a clinician's actual and desired practice for a specified measure, clinicians will be made aware of an unperceived learning need and will be motivated to make a change in their practice. ${ }^{1}$ While there are other commonly used terms for audit and feedback, it is our experience that some of these terms (e.g., report cards) can elicit negative reactions from clinicians. Audit and feedback may be given in various formats. ${ }^{2}$ Physicians may be provided with information about their performance relative to their peers, targets and/or benchmarks. Audit and feedback may include an action plan that can lead to improvement. ${ }^{3}$ Some may wonder why audit and feedback is necessary; physicians are

highly trained, motivated individuals seeking to provide the best care to their patients. Yet without receiving timely and specific practice feedback, it is difficult for physicians to identify unperceived learning needs or Framework ${ }^{4}$ presented by the Royal College encourages comes. When done properly, audit and feedback is both a professional development and quality improvement (QI) strategy. However, this strategy has to be designed and delivered in an effective manner and in line with the priorities of physicians and patients (Box 1$){ }^{5}$

At the next departmental meeting, Dr. Smith presented the idea of providing individual physicians with their practice data. Overall, there seemed to be a lot of interest around the concept, although a few of her colleagues questioned the utility of getting a "report card." Have other EDs done this and what is the evidence?

\section{What is the evidence to support audit and feedback?}

While several individual physician groups and healthcare regulatory bodies have embraced the use of audit and feedback for QI purposes, the effectiveness of audit and feedback can be variable. In the most recent Cochrane review, Ivers et al. analyzed 140 studies and found that audit and feedback leads to a median $4.3 \%$ absolute improvement in practice (interquartile range,

From the *University of Calgary, Department of Emergency Medicine, Calgary, AB; ${ }^{\dagger}$ McMaster University, Department of Medicine, Division of Emergency Medicine, Hamilton, ON; ${ }^{\ddagger}$ Saint Joseph's Healthcare Hamilton Charlton Campus, Department of Emergency Medicine, Hamilton, ON; ${ }^{\S}$ University of Toronto, Department of Medicine, Toronto, ON; ${ }^{\mathbb{I}}$ Saint Michael's Hospital, Toronto, Ontario, Canada; and the ${ }^{\#}$ University of Ottawa, Department of Emergency Medicine, Ottawa, ON.

Correspondence to: Dr. Shawn K. Dowling, Rockyview General Hospital - Holy Cross Ambulatory Care Centre, 5th Floor, Room 5A1057007, 14th St. SW, Calgary, AB T2V 1P9; Email: skdowling@gmail.com

(c) Canadian Association of Emergency Physicians 2020 
Box 1. Key elements of audit and feedback.

\section{Defining performance metrics}

a. Evidence-informed

b. Align data with goals and priorities of department/ physicians $^{7}$

c. Co-designed/selected by physicians/providers

d. When possible provide individual (rather than aggregate) data ${ }^{7}$

e. Metric should be within individual physician control

\section{Data report}

a. Include peer comparator or explicit goal/target ${ }^{7}$

b. Provide timely feedback over multiple instances ${ }^{7}$

c. Minimize extraneous cognitive load ${ }^{7}$

\section{Facilitation}

a. Facilitation (group or individual) can help

i. Address reactions to data and feedback process ${ }^{7}$

ii. Identify barriers and enablers to practice change

iii. Guide physicians in creating action plans

iv. Ensure the data are delivered both written and verbally ${ }^{7}$

v. Facilitation by a respected colleague not serving in a power position

4. Remeasurement to assess whether a change has occurred

$0.5 \%$ to $16 \%) .{ }^{6}$ However, the effect size was determined by the type of clinical behavior the audit and feedback aimed to address. Audit and feedback was more effective if baseline performance was low and feedback was: (1) from a supervisor or colleague, (2) provided more than once, (3) delivered in both written and verbal format, or and (4) included specific targets and an action plan. ${ }^{6}$

This suggests that audit and feedback is not a homogenous intervention, and the nature of the feedback and method of delivery influences its effectiveness. The best practices are further defined by Brehaut et al., ${ }^{7}$ who proposed 15 suggestions for optimizing feedback. This list includes recommendations on how to most effectively deliver practice feedback with respect to nature of the desired action, data available for feedback, how to display the data and deliver the feedback. ${ }^{7}$ How these concepts apply to the emergency medicine (EM) context will be explored in more detail throughout this study.

After reviewing the literature, Dr. Smith decided to use audit and feedback to improve the quality of care provided by the group. With the assistance of the hospital's information technology department, Dr. Smith was able to obtain access to several physician practice metrics, including number of patients seen per hour, imaging ordering rates, etc. How will she determine which data elements to use?

\section{What are common approaches to identifying audit and feedback indicators?}

Identifying which clinical indicators to provide physicians is a difficult yet critical step. A common starting point for selecting audit and feedback metrics for the $\mathrm{ED}$ is to use published clinical practice guidelines or achievable benchmarks of care (ABCs). ${ }^{8,9}$ Many audit and feedback interventions have used these guidelines and demonstrated clinical improvement. ${ }^{8}$ Another potential source of clinical indicators are the Choosing Wisely recommendations ${ }^{9}$ supported in a recent commentary by Ivers and Desveaux, outlining why audit and feedback is a fundamental element in reducing lowvalue care. ${ }^{10}$

An additional source of audit and feedback indicators may include making use of mandatory ED performance metrics. Although these may be viewed as "easy wins," there are important limitations to this approach. Metrics that are of interest to regulatory bodies may reflect departmental rather than individual physicians' performance. ${ }^{7}$ Examples of provincial performance metrics include Physician Initial Assessment (PIA) times and Ambulance Offload Times. An alternative would be to use these metrics to derive surrogate measures of individual clinician practice (i.e., report ED PIA to disposition times for clinicians).

Often, audit and feedback indicators with the greatest potential for improvement come from examination of local issues that are known to have significant variation in practice. One can also draw inspiration from variations identified in other similar departments. ED leaders should engage with broader EM communities to learn from those with audit and feedback expertise. 


\section{Co-designing metrics with physicians}

Although departments should use the above processes to derive some metrics to be used locally, we also advocate for a consensus process for the feedback on the final metrics to be used in the group. This can be done with a simple survey or a more robust Delphi process. ${ }^{11}$ This constitutes an important element of change management and provides each practitioner with the opportunity to opine on each metric. This collaborative approach is critical for engagement from frontline clinicians.

Physician groups looking to implement audit and feedback mechanisms could also establish objective evaluation criteria for current and newly proposed metrics according to the American College of Physicians criteria, ensuring only valuable metrics continue to be included (online Appendix A). Lastly, it is important to remember that improvement is a balance of the user's perception of value, capacity, and workload associated with the improvement task. ${ }^{12}$

\section{Dr. Smith decided to postpone dissemination of physician performance metrics, and instead formed a departmental working group to belp define their group's priorities. Together, they achieved consensus on areas of clinical care to focus on and began to define the data elements that would help them monitor those areas of care.}

\section{Aligning data with goals and priorities}

In the development of metrics, ED groups should consider the inclusion of measures that reflect current departmental goals. ${ }^{7}$ This may also include the modification of metrics to more closely align with these priorities and allows groups to quantify their individual and collective practice in areas that enable improvement. These specific metrics can often be found in hospital QI plans, or governing documents that set the improvement agenda for the organization.

Using available data, the working group decided on three areas of care to improve which each had a large variation in practice: patient flow, sepsis management, and computed tomography use. With these specific issues in mind, the working group developed the metrics for each area. The committee chose to review and report to individual physicians their data on: source of sepsis and time to initial lactate and antibiotics (see online Appendix A).

\section{Development of data reports}

The physician reports are a key element of audit and feedback, and thoughtful consideration should be given to the design and content. Although aggregate group data may be useful for system level measures, physician level feedback is generally more meaningful and is more likely to lead to change. Providing comparator data is beneficial as it helps support the desired behavior change. ${ }^{7}$ For metrics where an accepted ABC exists, providing this as a comparator can be more effective than peer data according to one study. In one study, including a behavior change message (i.e., message that supports desired action) resulted in fewer antibiotic prescriptions compared with control. ${ }^{13}$

The optimal frequency of distributing reports is unknown and may depend on the data being provided. Providing feedback in a timely manner is important to ensure the data is relevant and actionable. ${ }^{7}$ Exceedingly frequent data delivery may lead to data fatigue or dramatic practice changes based on a small number of cases. $^{7}$ By contrast, infrequent feedback may be discounted as no longer relevant due to a perceived change in personal practice that may or may not have occurred. Ideally, physicians would individually track their performance over time and see if their action plans resulted in noticeable improvements. Data reports can include a trend-over-time graphic to allow for comparison to prior performance.

When creating the reports, it is important to reduce extraneous cognitive load for feedback recipients. ${ }^{7}$ This may mean providing fewer metrics or only data for the most relevant indicators. One should also simplify the data presented. If the data require significant effort to interpret, it may be misunderstood or entirely ignored. Reports should be presented both verbally and in writing, and figures should be consistent with the message being conveyed (e.g., poor performance at bottom of a graph v. good performance at top of a graph).

Ensuring reports are easily accessible and actively disseminated will increase engagement among physicians. Passive report dissemination (e.g., physicians needing to retrieve the report from an online dashboard) may be a barrier to physicians. Without context, physicians may question the validity of the data, thus acknowledging the limitations of the data at the outset is critical.

Finally, a stepwise approach in establishing the scope and progressive rollout of performance metrics is important. Careful consideration must be given to the 


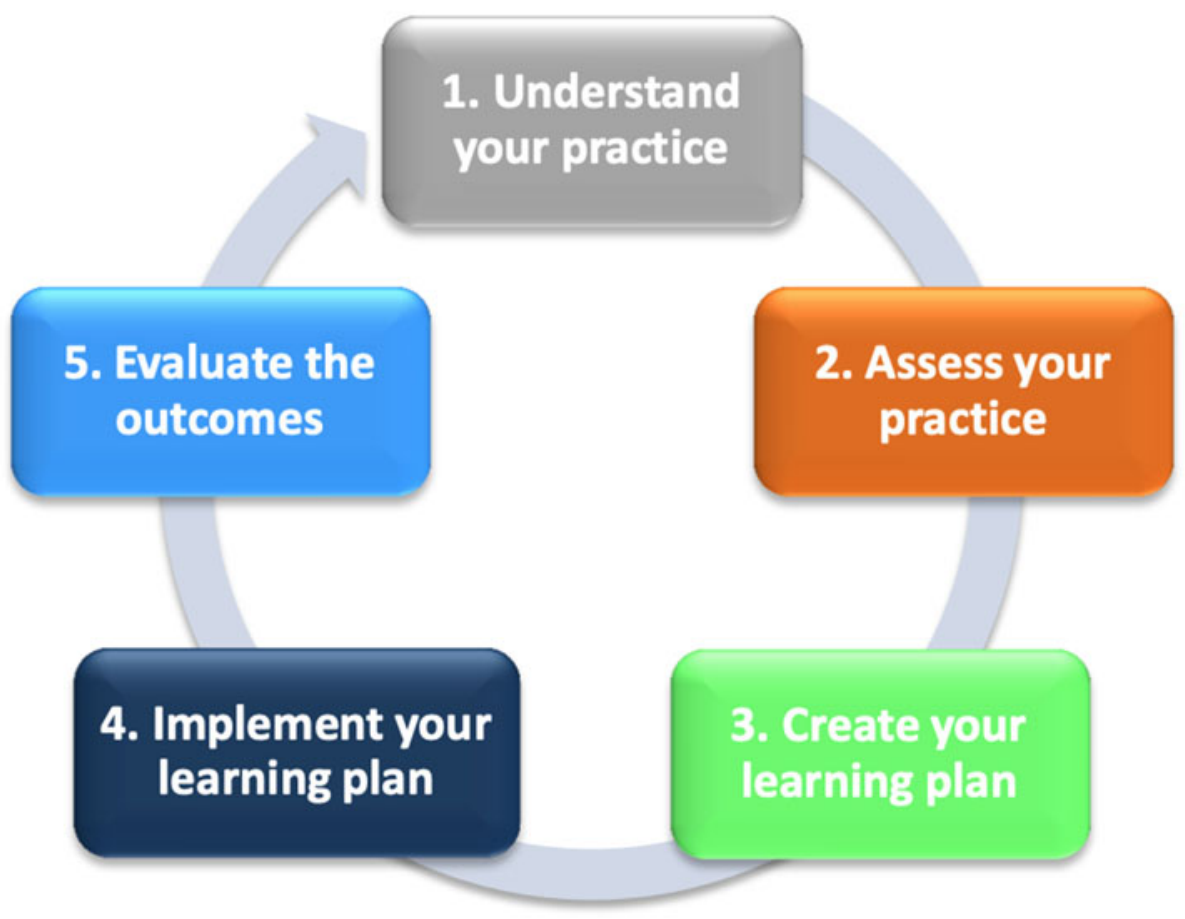

Figure 1. Physician practice improvement.

initial set of measures that are proposed within a group so as to ensure a balanced approach. As an example, it could be dangerous to implement an initial set of measures which included time to consult, advanced imaging use rates, number of patients seen per hour, and average billings per patient. This suite would incentivize the rapid cycling of patients with fewer investigations and more procedures. This "directionality" of initial performance metrics must include balancing measures from the outset such as percent consults admitted and 72 hours ED return and admit rates. ${ }^{14}$

As the working group began to finalize a mock-up of a performance report card, Dr. Smith wondered about the logistics of presenting these metrics: Do I send them to the group by email or do I meet with each individual?

\section{How to deliver and facilitate audit and feedback?}

To maximize the impact of audit and feedback on individual clinicians, we must also consider emerging trends in medical education and professional development. Recently, the Royal College of Physicians and Surgeons of Canada has made audit and feedback an integral part of their Competency By Design Framework, emphasizing the importance of Physician Practice Improvement
(Figure 1). ${ }^{4}$ To do this successfully, we need to provide clinicians with their practice data as well as provide them with the tools to implement action plans. This process requires more than just passive dissemination of physician data.

Group facilitated feedback sessions have been used in audit and feedback sessions to leverage social learning theory and promote discussion among clinicians ${ }^{15}$ (Figure 2). In these sessions, participants go through a predictable pattern of reacting to and understanding the data, contextualizing, reflecting, and planning for change. ${ }^{16}$ This process helps physicians convert their practice data into meaningful information that can be used for practice improvement. We have also seen a growing call for medical coaching in the mainstream media, with multiple articles published on the topic. ${ }^{17-19}$ This high intensity activity will require formal assessment to establish its role in physician practice improvement.

\section{Additional considerations: privacy and controversies related to audit and feedback}

A constant tension exists as to whether audit and feedback should be used as a performance management tool or as a self-reflective strategy. Although arguments can be made on both fronts, the current data 


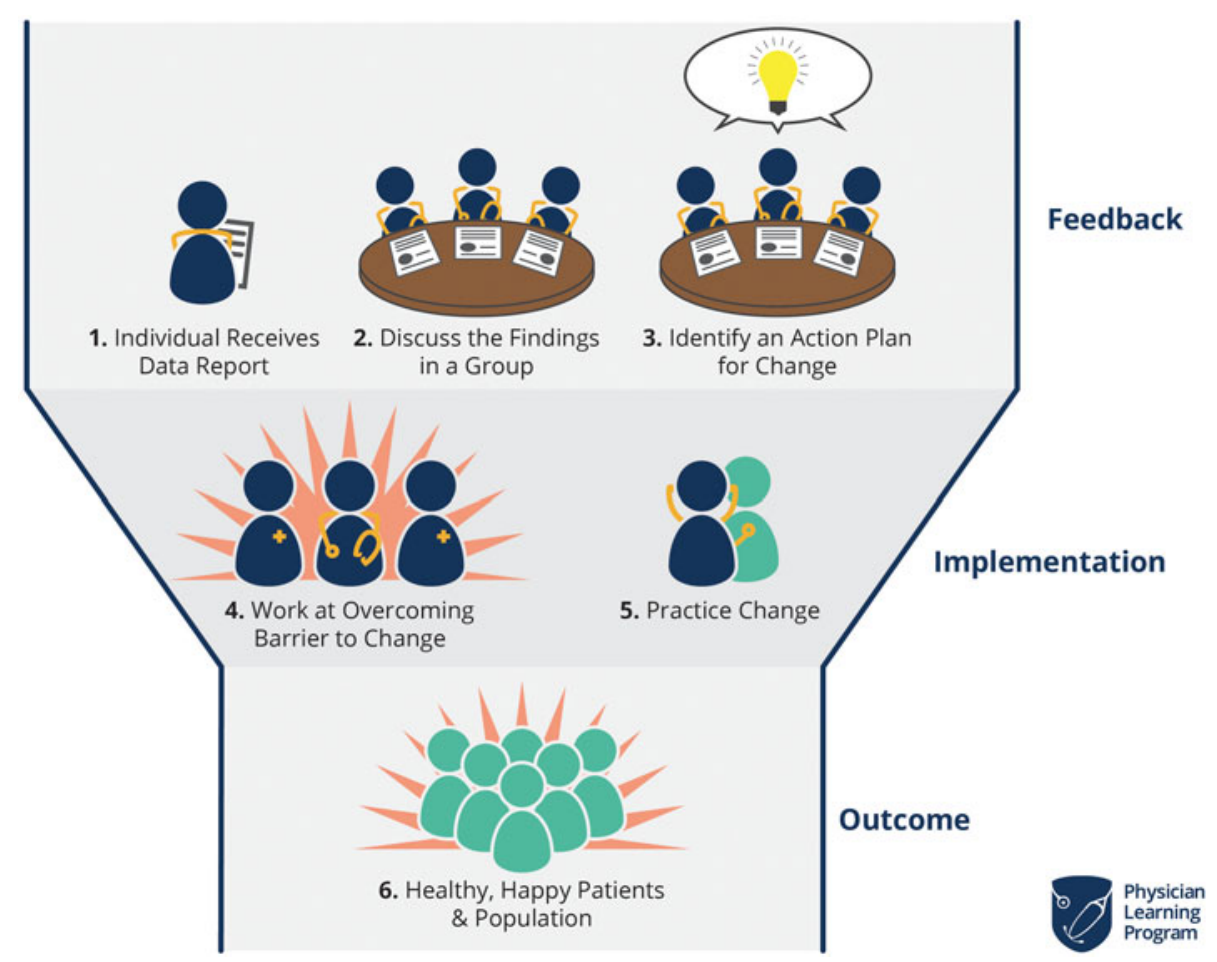

Figure 2. Calgary audit and feedback framework.

available are unable to provide a holistic view of the "quality of care that one physician provides," at best, we can provide snapshots of a physician practice with respect to the metrics provided. It is important to have real discussions about the permissibility of the data and the extent to which can be used within a group. Clear evidence of high-risk practice may require early intervention that should be discussed at the outset. As such, using audit and feedback for performance assessment, pay for performance, or employment decisions should be done with caution until there is evidence around which strategy is most effective. Yet, it is also easy to imagine the use of audit and feedback to establish minimum practice metrics or envision their use in a punitive sense. This is often dubbed "data for accountability." Within this publication, we openly advocate for use of this audit and feedback as a self-reflective professional development tool.

As with any data, those individuals collecting the data are responsible for its privacy. Although there is often an interest in providing unblinded data reports (whereby physicians are openly compared with each other); this strategy has not been studied and could have detrimental impact on morale and gaming of metrics to improve ones' rank within the department. How the de-identified physician data are disseminated should be discussed within the group, which includes specifics on data storage, how the data will be used, and which individuals within the department should have access to identifiable datasets. Furthermore, groups should explicitly state their policy toward dissemination of de-identified data outside of the clinical group (i.e., hospital leadership) to ensure the data are used for its intended purpose.

As a result of physician inclusion, a model for audit and feedback is developed. Based on this, Dr. Smith has designed a feedback plan that includes scheduling small group discussions with peers. She also began to develop coaching modules for her physicians. Within the first 3 months of implementing the audit and feedback program, she sees modest but encouraging results within the group. She will use feedback to further improve the process.

\section{CONCLUSION}

Audit and feedback can be a powerful QI tool that should be considered by EM practitioners and EDs. To maximize its potential, careful design of its metrics and a 
deliberate implementation process should be undertaken. Particularly, the processes should be physician-led, align with departmental and patient-centered priorities, and incorporate the best possible evidence on both clinical care and the tenets of audit and feedback. The individual data that result from audit and feedback activities should be used for data-driven practice improvement as part of continuous professional development activities. This process should also be mobilized as a tool to decrease lowvalue care and improve the quality of care provided in the ED.

Keywords: Administration, quality improvement, safety

Supplementary material: The supplementary material for this article can be found at https://doi.org/10.1017/cem.2020.28.

\section{REFERENCES}

1. Meyer and, Singh H. The path to diagnostic excellence includes feedback to calibrate how clinicians think. $7 A M A$ 2019;373(26):2493-5. doi:10.1001/jama.2019.0113.

2. Jamtvedt G, Young JM, Kristoffersen DT, O'Brien MA, Oxman AD. Audit and feedback: effects on professional practice and health care outcomes. Cochrane Database Syst Rev 2006; (2):CD000259. doi:10.1002/14651858.CD000259.pub2.

3. Ivers NM, Sales A, Colquhoun H, et al. No more "business as usual" with audit and feedback interventions: towards an agenda for a reinvigorated intervention. Implement Sci 2014;9:14. doi:10.1186/1748-5908-9-14.

4. The Royal College of Physicians and Surgeons of Canada. Coaching and CBD. Royal College of Physicians and Surgeons of Canada website. http://www.royalcollege.ca/rcsite/ cbd/implementation/wbas/coaching-and-cbd-e (accessed March 19, 2020).

5. MacLean CH, Kerr EA, Qaseem A. Time out - charting a path for improving performance measurement. $N$ Engl 7 Med 2018;378(19):1757-61. doi:10.1056/NEJMp1002530.

6. Ivers N, Jamtvedt G, Flottorp S, et al. Audit and feedback: effects on professional practice and healthcare outcomes. Cochrane Database Syst Rev 2012;6(6):CD000259. doi:10.1002/14651858.CD000259.pub3.

7. Brehaut JC, Colquhoun HL, Eva KW, et al. Practice feedback interventions: 15 suggestions for optimizing effectiveness. Ann Intern Med 2016;164(6):435-41. doi:10.7326/M15-2248.
8. Kiefe CI, Allison JJ, Williams OD, Person SD, Weaver MT, Weissman NW. Improving quality improvement using achievable benchmarks for physician feedback: a randomized controlled trial. FAMA 2001;285(22):2871-9. doi:10.1001/ jama.285.22.2871.

9. Reyes M, Paulus E, Hronek C, et al. Choosing wisely campaign: report card and achievable benchmarks of care for children's hospitals. Hosp Pediatr 2017;7(11):633-41. doi:10.1542/hpeds.2017-0029.

10. Ivers NM, Desveaux L. De-implementation of low-value care: use audit and feedback wisely. Healthc Q 2019;18(1):41-7.

11. Dalkey N. An experimental study of group opinion: the Delphi method. Futures 1969;1(5):408-26. doi:10.1016/ S0016-3287(69)80025-X.

12. Hayes CW, Goldmann D. Highly adoptable improvement: a practical model and toolkit to address adoptability and sustainability of quality improvement initiatives. $7 \mathrm{t}$ Comm 7 Qual Patient Saf 2018;44(3):155-63. doi:10.1016/j.jcjq.2017. 09.005 .

13. Elouafkaoui P, Young L, Newlands R, et al. An audit and feedback intervention for reducing antibiotic prescribing in general dental practice: The RAPiD Cluster Randomised Controlled Trial. PLoS Med 2016;13(8):e1002115. doi:10.1371/journal.pmed.1002115.

14. The Institute for Healthcare Improvement (IHI). Science of improvement: establishing measures. http://www.ihi.org/ offerings/MembershipsNetworks/collaboratives/Pages/ default.aspx (accessed July 18, 2019).

15. Cooke LJ, Duncan D, Rivera L, Dowling SK, Symonds C, Armson H. The Calgary Audit and Feedback Framework: a practical, evidence-informed approach for the design and implementation of socially constructed learning interventions using audit and group feedback. Implement Sci 2018;13 (1):136. doi:10.1186/s13012-018-0829-3.

16. Cooke LJ, Duncan D, Rivera L, Dowling SK, Symonds C, Armson $\mathrm{H}$. How do physicians behave when they participate in audit and feedback activities in a group with their peers? Implement $\mathrm{Sci}$ 2018;13(1):104. doi:10.1186/s13012-018-0796-8.

17. Watling C, Driessen E, van der Vleuten CP, Vanstone M, Lingard L. Music lessons: revealing medicine's learning culture through a comparison with that of music. Med Educ 2013;47(8):842-50. doi:10.1111/medu.12235.

18. Watling C, Driessen E, van der Vleuten CP, Lingard L. Learning culture and feedback: an international study of medical athletes and musicians. Med Educ 2014;48(7):71323. doi:10.1111/medu. 12407 .

19. Watling CJ, LaDonna KA. Where philosophy meets culture: exploring how coaches conceptualise their roles. Med Educ 2019;53:467-76. doi:10.1111/medu.13799. 